Check for updates

Cite this: Mater. Chem. Front., 2019, 3, 1888

Received 13th March 2019, Accepted 17th July 2019

DOI: 10.1039/c9qm00150f

rsc.li/frontiers-materials

\section{Directional self-assembly of gold nanorods into 1D and 2D arrays by quadruple hydrogen bonding $\dagger$}

\author{
Mingming Zhai, $\ddagger^{\mathrm{a}}$ Qiang Chen, $\ddagger^{\mathrm{a}}$ Wei Yuan, ${ }^{\mathrm{a}}$ Qixun Shi (DD *ab and Hui Xu (DD *a
}

A facile, bottom-up technique is developed to produce highly ordered end-to-end and side-by-side assembly of gold nanorods (GNRs) via quadruple hydrogen bonding. The switching between end-to-end and side-by-side aggregation modes can be achieved by tuning the crosslinker concentration.
Owing to their special optical and electronic properties, GNRs have gained increasing attention over the past few decades., Controlled assembly of GNRs into specific arrangements further grants them size- and shape-dependent properties that are different from the individual GNRs and they have significant applications in sensing, optical and optoelectronic devices and biomedicine. ${ }^{3-6}$ The self-assembly of GNRs thus represents one of the research hotspots of the field of nanoparticles in recent years. Various supramolecular assembly strategies of GNRs have been developed based on noncovalent intermolecular interactions between ligands containing a thiol or disulfide group at one end to form the $\mathrm{Au}-\mathrm{S}$ bonds and other functional groups at the other end for supramolecular interactions. ${ }^{2,5,7-11}$ To date, among all noncovalent interactions available, hydrophobic forces, ${ }^{12-14}$ electrostatic forces, ${ }^{15-17}$ host-guest interactions, ${ }^{18-22}$ and coordination bonds ${ }^{23,24}$ have been researched in great detail. However, the application of hydrogen bonding (H-bonding) has been demonstrated in only a few cases. ${ }^{25-28}$ Considering the high practical utility and wide availability of $\mathrm{H}$-bonding arrays in the supramolecular chemistry field, we sought to develop a new assembly strategy of GNRs based on this non-covalent interaction, especially in much less explored competing polar aqueous media. The 2-ureido-4[1 $\mathrm{H}]$-pyrimidinone (UPy) introduced by Meijer and coworkers as a quadruple hydrogen bonding building unit has been routinely applied in the field of supramolecular organic polymers due to its simplicity in design, easy synthesis, extremely strong association constant $\left(K_{\mathrm{dim}}>10^{7} \mathrm{M}^{-1}\right)$ compared with

\footnotetext{
${ }^{a}$ Institute of Advanced Synthesis, School of Chemistry and Molecular Engineering, Jiangsu National Synergetic Innovation Center for Advanced Materials, Nanjing. Tech University, Nanjing 211816, China. E-mail: ias_qxshi@njtech.edu.cn, ias_hxu@njtech.edu.cn

${ }^{b}$ State Key Laboratory of Fine Chemicals, Dalian University of Technology, Dalian 116024, China

$\dagger$ Electronic supplementary information (ESI) available: Experimental details, and TEM images. See DOI: 10.1039/c9qm00150f

\$ These two authors contributed equally to this work.
}

double and triple hydrogen bonding, and self-assembly ability through UPy-UPy dimerization which apparently reduces the synthesis efforts. ${ }^{29,30}$ All the above attractive features of the UPy unit make it an excellent candidate for probing the H-bonding based assembly of GNRs. In this paper, for the first time we present a facile, bottom-up technique that produces highly ordered arrays of colloidal GNRs utilizing thiol-functionalized UPy derivative 1 (Fig. 1) as a crosslinker operating through quadruple hydrogen bonding self-assembly. Our findings reveal that by tuning the crosslinker concentration, two different GNR assembly modes can be achieved. The switching between end-toend and side-by-side aggregation of GNRs was demonstrated at low and high linker concentrations in solution, respectively.

The parent GNRs coated with hexadecyltrimethylammonium bromide (CTAB) were synthesized using a previously reported seed-mediated technique. ${ }^{31}$ A typical transmission electron microscopy (TEM) image of the GNRs of average aspect ratio 5.0 (average length $50 \pm 4 \mathrm{~nm}$; average diameter $10 \pm 2 \mathrm{~nm}$ ) is shown in Fig. S2 (ESI $\dagger$ ). Both the size and shape of the GNRs were nearly uniform. It showed that CTAB-coated GNRs did not self-assemble in the absence of the crosslinker 1. These GNRs remained randomly dispersed when dried on a TEM grid. After introduction of a controlled amount of thiol-terminated Upy crosslinker 1 into the solution of GNRs, selective end-to-end

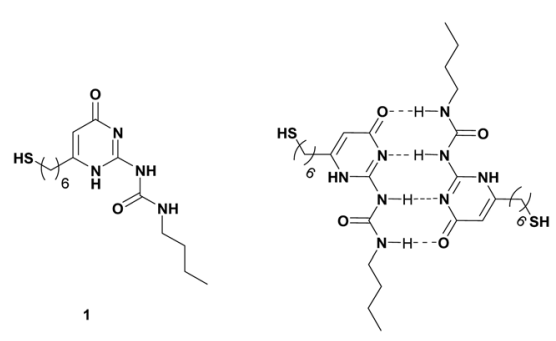

Fig. 1 Structure of thiol-functionalized UPy derivative 1 and the formation of the UPy dimers by quadruple hydrogen bonding. 


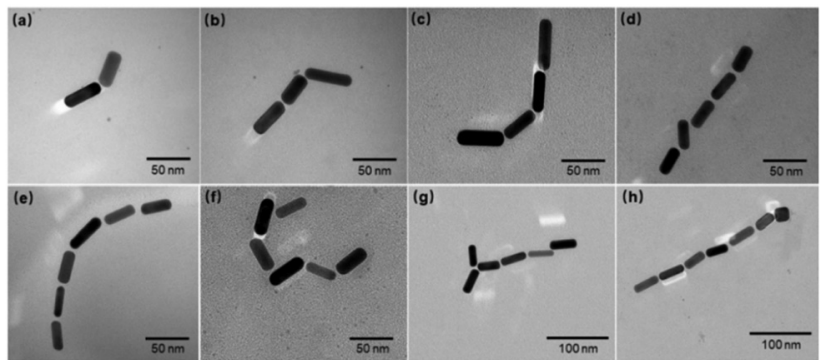

Fig. 2 Representative TEM images depicting the alignment of GNRs at $2.3 \mathrm{mM}$ of crosslinker 1. (a) Dimer, (b) trimer, (c) tetramer, (d) pentamer, $(\mathrm{e}-\mathrm{g})$ bent, irregular, and branched hexamer, and (h) heptamer

assembly was observed. As shown in Fig. 2, at low concentration of crosslinker 1 (2.3 $\mathrm{mM})$, a varying number of aligned GNR arrangements were observed over the dried TEM grid, including dimers (Fig. 2a), trimers (Fig. 2b), tetramers (Fig. 2c), pentamers (Fig. 2d), hexamers (Fig. 2e-g), and heptamers (Fig. 2h). Among them, linear, quasi-linear, bent, irregular and branched structures were frequently observed. Importantly, the structures share the common characteristic that nearly all the GNRs are linked by end-to-end aggregation and the assemblies obtained are highly ordered. Low magnification TEM images depicting the alignment of GNRs at $2.3 \mathrm{mM}$ of crosslinker 1 are shown in Fig. S3 (ESI $\dagger$ ). Side-by-side assembly was hardly ever observed at this low concentration of crosslinker $\mathbf{1}$.

With the increase of crosslinker concentration, the aligned GNR chains grow longer. As shown in Fig. 3, at a crosslinker concentration of $4.6 \mathrm{mM}$, the number of GNR units in linear end-to-end assemblies increases up to 10-17. This is due to the fact that the number of crosslinkers $\mathbf{1}$ at the two ends of the GNRs increases, and the cooperative quadruple hydrogen bonding induced by the very rigid Upy linker makes the alignment straight linear rather than irregular or branched. ${ }^{32}$ Formation of a small amount of side-by-side GNR aggregates was also observed at this concentration. This can be explained by simultaneous replacement of adsorbed cationic CTAB molecules on the side wall of the GNRs by the crosslinker 1, resulting in side-by-side assembly induced by H-bonding.

As the concentration of crosslinker 1 was further increased, a larger fraction of GNRs was assembling and the resulting $2 \mathrm{D}$ array of nanoparticles grew in size. Fig. 4a shows a typical

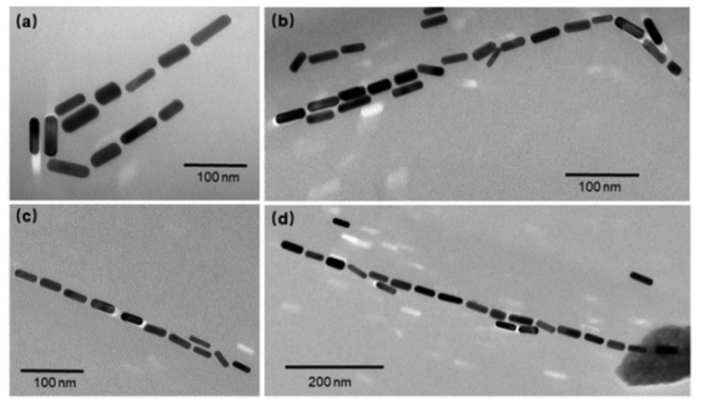

Fig. 3 Representative TEM images of GNR end-to-end assemblies by increasing the concentration of crosslinker 1 to $4.6 \mathrm{mM}$.
2D ordering within a monolayer of GNRs formed in the presence of $9.2 \mathrm{mM}$ crosslinker 1. Both end-to-end and sideby-side assemblies coexist at this concentration. As the concentration of crosslinker 1 increased further, multilayers of GNRs started to form (Fig. 4b-d). Fig. 4e and f show TEM images taken from the assembly formed at a crosslinker concentration of $15.3 \mathrm{mM}$. 2D ordered structures were observed that spread over the whole image $\left(\sim 6 \mu \mathrm{m}^{2}\right.$ area) in which GNRs are arranged dominantly side-by-side. A small fraction of perpendicular arrangement of GNRs (Fig. 4f) was also observed together with side-by-side assembled superstructures (Fig. 4e). A large area of circular dots was seen from a longitudinal direction indicating that GNRs attached perpendicularly to the substrate. At a higher concentration of crosslinker 1, the adsorbed cationic CTAB molecules were effectively replaced resulting in a full coverage (both ends and sides) of the GNRs with UPy-SH linkers. Under high concentration regime, side-by-side GNR assemblies were dominant because there were more linkers on the GNR sides than on the GNR ends, resulting in a stronger network of H-bonds between the GNR sides than between the GNR ends. Moreover, AFM measurement was conducted to further confirm the 2D multilayer arrays at a crosslinker concentration of $12.3 \mathrm{mM}$ (Fig. S4a, ESI $\dagger$ ). The height of the assembled superstructure is observed to be about $33.6 \mathrm{~nm}$ (Fig. S4b, ESI $\dagger$ ), which is approximately 3-4 times the diameter of individual GNRs, indicating that this 2D array assembly structure consists of 4 layers of GNRs. It should be noted that the control experiment with CTAB coated GNRs at very high concentration in the absence of crosslinker 1 resulted in non-ordered aggregation of GNRs with only one layer (Fig. S5, ESI $\dagger$ ). This reveals that the well-ordered end-to-end and side-by-side assemblies shown in Fig. 4 are triggered by crosslinker 1 .

We found that depending on the crosslinker concentration in solution, GNRs assemble through two different attachment modes: end-to-end attachment and side-by-side attachment. We speculate that these two assembly modes were closely related to the affinity of the $\mathrm{Au}-\mathrm{S}$ binding and the repulsion between the cationic CTAB molecules on the side. ${ }^{33}$ Crosslinker 1 has two functional groups, a thiol group and a 2-ureido-4 $(1 H)$-pyrimidinone moiety,

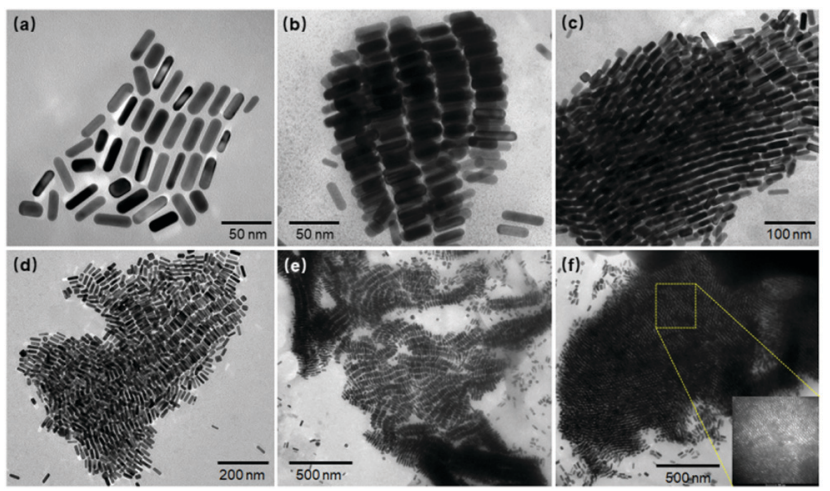

Fig. 4 Representative TEM images of GNR assemblies at different concentrations of crosslinker 1. (a) $9.2 \mathrm{mM},(\mathrm{b}-\mathrm{d}) 12.3 \mathrm{mM}$, and (e and f) $15.3 \mathrm{mM}$. The inset in ( $\mathrm{f}$ ) shows perpendicular arrangement of GNRs. 


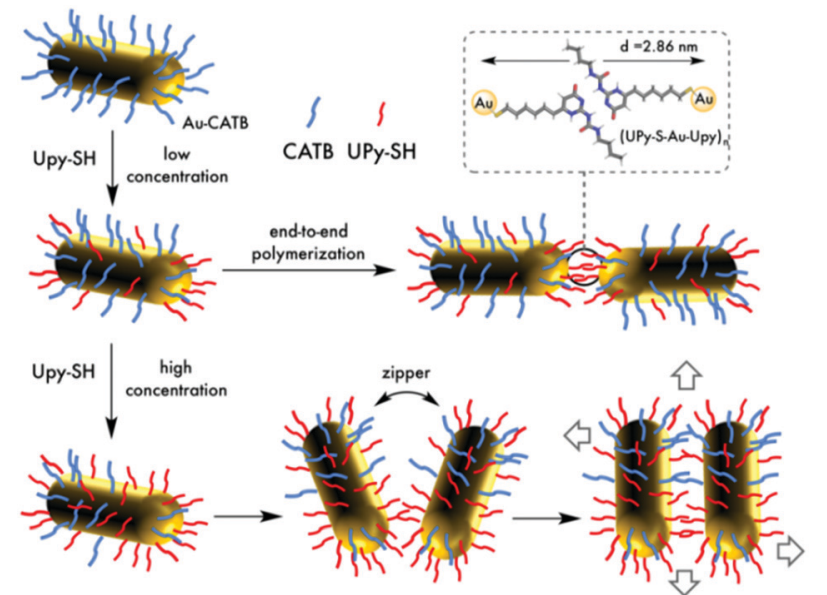

Fig. 5 Schematic representation of end-to-end and side-by-side assembly modes of GNRs.

and both can form bonds with Au. Bonding between the thiol moiety and $\mathrm{Au}$ is more favored because of the strong $\mathrm{Au}-\mathrm{S}$ covalent bond formation. The linkers can connect two adjacent GNRs via quadruple hydrogen bonding, as illustrated in Fig. 5. With the addition of crosslinker 1, the thiol groups adsorb preferentially onto loosely covered ends of GNRs (i.e., the $\{111\}$ $\mathrm{Au}$ facets) because of preferential binding of CTAB along the $\{100\}$ facet of the longitudinal side. Whereafter, the excess linker would subsequently bind to the side surface or even replace the cationic CTAB molecules. Under low coverage conditions the electrostatic repulsion between the positively charged CTAB molecules prevents side-by-side aggregation and thus, end-to-end attachment is predominant. In contrast, at a higher linker concentration, the fraction of crosslinker 1 starts to increase also on the sides of the GNRs and eventually overcomes the electrostatic repulsion between the CTAB molecules. At this point it is not clear whether replacement of CTAB molecules is taking place at high concentration of the linker or merely the aggregation is caused by an increase in the number of Upy-SH linkers attached to the GNRs. Most likely, the formation of sufficiently large clusters of Upy-SH molecules is required to ensure the cooperative interaction of two nanoparticles (zipper-effect) resulting in the linker concentration threshold under which only end-to-end assembly is observed. We also conducted two control experiments on crosslinker 2 with double hydrogen bonding, and crosslinker 3 and $\mathbf{4}$ which have complementary triple hydrogen bonding units (structures see Fig. S1, ESI $\dagger$ ). At a low crosslinker concentration, a few short oligomers were formed, as shown in Fig. S6 and S7 (ESI $\dagger$ ). However, long and linear aligned GNR chains were not observed. In addition, using either 2 or $\mathbf{3}$ and $\mathbf{4}$ as crosslinkers, apparent 2D ordered aggregates have not been observed in the TEM images under higher concentrations (Fig. S6 and S7, ESI $\dagger$ ).

In order to confirm that the GNR alignment is indeed due to hydrogen bonding, we measured the spacing between the neighboring GNRs. The average value was estimated from 100 interparticle distances in the TEM image and the histogram was prepared (Fig. S8, ESI $\dagger$ ). The distribution of the pairwise spacing between the attached ends of GNRs reveals the peak centered at $2.8 \pm 0.3 \mathrm{~nm}$. The predicted molecular length of two crosslinker molecules based on the molecular model together with the hydrogen bond length is $\sim 2.86 \mathrm{~nm}$. The top peak at $2.8 \mathrm{~nm}$ is thus fully consistent with the proposed model where two linkers connect the GNRs through H-bonding.

\section{Conclusions}

In conclusion, well-ordered end-to-end and side-by-side assembly of GNRs via quadruple hydrogen bonding was designed and experimentally demonstrated by TEM measurements. The uniaxial self-assembly of the GNRs was due to the selective binding of crosslinker molecules at the two ends of the GNRs and the quadruple intermolecular hydrogen bonding between the GNRbound thiol-terminated Upy crosslinkers. The switching between end-to-end and side-by-side GNR aggregation modes can be induced by tuning the concentration of crosslinker 1 . As the amount of crosslinker 1 increases, self-assembly of GNRs changes from end-to-end to side-by-side mode. Importantly, the application of H-bonding to drive the assembly of GNRs in highly competing water media was successfully demonstrated. The uniform and predetermined assembly of GNRs with strong and directional electromagnetic enhancements offers a strong confinement of light within nanoscale gaps, hence enabling their future exciting applications in nanoelectronics and plasmonics.

\section{Conflicts of interest}

There are no conflicts to declare.

\section{Acknowledgements}

The authors thank Prof. Edvinas Orentas for the helpful discussion and construction of the molecular model of a UPy dimer. We are grateful for the financial support from the Natural Science Foundation of Jiangsu Province (BK20181375 and BK20170977), Nanjing Tech University (Start-up Grant 39837129 and 39837122) and the State Key Laboratory of Fine Chemicals (KF1707). We are also thankful for the financial support from SICAM Fellowship from Jiangsu National Synergetic Innovation Center for Advanced Materials.

\section{Notes and references}

1 H. Chen, L. Shao, Q. Li and J. Wang, Chem. Soc. Rev., 2013, 42, 2679-2724.

2 X. Huang, S. Neretina and M. A. El-Sayed, Adv. Mater., 2009, 21, 4880-4910.

3 M. Hu, J. Chen, Z.-Y. Li, L. Au, G. V. Hartland, X. Li, M. Marquez and Y. Xia, Chem. Soc. Rev., 2006, 35, 1084-1094.

4 M. R. Jones, K. D. Osberg, R. J. Macfarlane, M. R. Langille and C. A. Mirkin, Chem. Rev., 2011, 111, 3736-3827.

5 L. Vigderman, B. P. Khanal and E. R. Zubarev, Adv. Mater., 2012, 24, 4811-4841. 
6 J. Zhou, Z. Cao, N. Panwar, R. Hu, X. Wang, J. Qu, S. C. Tjin, G. Xu and K.-T. Yong, Coord. Chem. Rev., 2017, 352, 15-66.

7 L. Xu, H. Kuang, L. Wang and C. Xu, J. Mater. Chem., 2011, 21, 16759-16782.

8 K. Liu, N. Zhao and E. Kumacheva, Chem. Soc. Rev., 2011, 40, 656-671.

9 K. K. Caswell, J. N. Wilson, U. H. F. Bunz and C. J. Murphy, J. Am. Chem. Soc., 2003, 125, 13914-13915.

10 J.-Y. Chang, H. Wu, H. Chen, Y.-C. Ling and W. Tan, Chem. Commun., 2005, 1092-1094.

11 F. C.-M. Leung, S. Y.-L. Leung, C. Y.-S. Chung and V. W.-W. Yam, J. Am. Chem. Soc., 2016, 138, 2989-2992.

12 Z. Nie, D. Fava, E. Kumacheva, S. Zou, G. C. Walker and M. Rubinstein, Nat. Mater., 2007, 6, 609-614.

13 Z. Nie, D. Fava, M. Rubinstein and E. Kumacheva, J. Am. Chem. Soc., 2008, 130, 3683-3689.

14 Y. Wang, A. E. DePrince, III, S. K. Gray, X.-M. Lin and M. Pelton, J. Phys. Chem. Lett., 2010, 1, 2692-2698.

15 P. K. Sudeep, S. T. S. Joseph and K. G. Thomas, J. Am. Chem. Soc., 2005, 127, 6516-6517.

16 S. Zhang, X. Kou, Z. Yang, Q. Shi, G. D. Stucky, L. Sun, J. Wang and C. Yan, Chem. Commun., 2007, 1816-1818.

17 L. Zhong, X. Zhou, S. Bao, Y. Shi, Y. Wang, S. Hong, Y. Huang, X. Wang, Z. Xie and Q. Zhang, J. Mater. Chem., 2011, 21, 14448-14455.

18 H. Nakashima, K. Furukawa, Y. Kashimura and K. Torimitsu, Chem. Commun., 2007, 1080-1082.
19 S. T. Jones, J. M. Zayed and O. A. Scherman, Nanoscale, 2013, 5, 5299-5302.

20 D. Lin, J. Wu, H. Ju and F. Yan, Biosens. Bioelectron., 2013, 45, 195-200.

21 Y. Xu, X. Wang and X. Ma, Dyes Pigm., 2017, 144, 168-172.

22 Y. Xu, X. Wang and X. Ma, Dyes Pigm., 2017, 145, 385-390.

23 Y.-T. Chan, S. Li, C. N. Moorefield, P. Wang, C. D. Shreiner and G. R. Newkome, Chem. - Eur. J., 2010, 16, 4164-4168.

24 P. R. Selvakannan, E. Dumas, F. Dumur, C. Péchoux, P. Beaunier, A. Etcheberry, F. Sécheresse, H. Remita and C. R. Mayer, J. Colloid Interface Sci., 2010, 349, 93-97.

25 K. G. Thomas, S. Barazzouk, B. I. Ipe, S. T. S. Joseph and P. V. Kamat, J. Phys. Chem. B, 2004, 108, 13066-13068.

26 X. Hu, W. Cheng, T. Wang, E. Wang and S. Dong, Nanotechnology, 2005, 16, 2164-2169.

27 W. Ni, R. A. Mosquera, J. Pérez-Juste and L. M. Liz-marzán, J. Phys. Chem. Lett., 2010, 1, 1181-1185.

28 S. F. Tan, U. Anand and U. Mirsaidov, ACS Nano, 2017, 11, 1633-1640.

29 F. H. Beijer, R. P. Sijbesma, H. Kooijman, A. L. Spek and E. W. Meijer, J. Am. Chem. Soc., 1998, 120, 6761-6769.

30 T. F. A. De Greef, M. M. J. Smulders, M. Wolffs, A. P. H. J. Schenning, R. P. Sijbesma and E. W. Meijer, Chem. Rev., 2009, 109, 5687-5754.

31 A. Gole and C. J. Murphy, Chem. Mater., 2004, 16, 3633-3640. 32 P. Pramod and K. G. Thomas, Adv. Mater., 2008, 20, 4300-4305.

33 J. Liu, C. Kan, Y. Li, H. Xu, Y. Ni and D. Shi, Appl. Phys. Lett., 2014, 104, 253105. 